Research Report

\title{
Generalization of seizures parallels the formation of "dark" neurons in the hippocampus and pontine reticular formation after focal-cortical application of 4-aminopyridine (4-AP) in the rat
}

\author{
Péter Baracskay, Zsuzsanna Szepesi, Gergely Orbán, Gábor Juhász, András Czurkó* \\ Laboratory of Proteomics, Institute of Biology, Faculty of Natural Sciences, Eötvös Loránd University, H-1117 Budapest, Hungary
}

\section{A R T I C L E I N F O}

Article history:

Accepted 13 June 2008

Available online 21 June 2008

Keywords:

Epilepsy

Dark neuron

Hippocampus

Pontine reticular formation

Cell injury

Animal model

Neurogliaform cell

Astrocyte

Status epilepticus

\begin{abstract}
A B S T R A C T
Distribution and time course of the occurrence of "dark" neurons were compared with the EEG activity and behavior of rats during 4-aminopyridine (4-AP) induced epileptic seizures. A crystal of the $\mathrm{K}^{+}$channel blocker 4-AP $(0.5 \mathrm{mg} / \mathrm{kg})$ was placed onto the exposed parietooccipital cortex of Halothane-anesthetized rats for $40 \mathrm{~min}$. Thereafter, the anesthesia was discontinued and the behavioral signs of the epileptic seizure activity were observed. The presence of "dark" neurons was demonstrated by the sensitive silver method of Gallyas in rats sacrificed at 0, 3 and $6 \mathrm{~h}$ after the end of the 4-AP crystal application. The EEG activity was recorded in the rats with longer survival times. The EEG analysis revealed the generalization of the epileptic seizures. We found that the formation of "dark" neurons in the hippocampus and the pontine reticular formation paralleled the generalization of the seizures.
\end{abstract}

(c) 2008 Elsevier B.V. All rights reserved.

\section{Introduction}

Understanding of the development and generalization of the epileptic seizure activity and the accompanying changes in the molecular structure of the brain cells are great challenges in neurology and neuroscience. It is well known that epileptic convulsions are the end-result of the hyperexcitability of several excitatory neuronal circuits and of the spread of the seizure activity over the whole cerebrum. In addition to the excitatory circuits, the inhibitory interneuron networks are also involved in the progression of epileptic seizure activity (Benardo, 1997; Mody et al., 1992). The epileptic activity causes sustained depolarization in both excitatory and inhibitory neurons with a concomitant elevation of intracellular $\mathrm{Ca}^{++}$, in turn, results in both pathological and protective changes in the cellular protein composition (Ogita et al., 2005).

The seizure-related, significantly elevated intracellular $\mathrm{Ca}^{++}$-concentration is a cellular stress which, on the morphological level, can be manifested in the compaction of the ultrastructure of neurons. Such neurons are traditionally

\footnotetext{
* Corresponding author. Laboratory of Proteomics, Institute of Biology, Faculty of Natural Sciences, Eötvös Loránd University, Pázmány Péter sétány. 1/c, H-1117 Budapest, Hungary. Fax: +3613812204.

E-mail addresses: czurko2@yahoo.com, aczurko@caesar.elte.hu (A. Czurkó).

Abbreviations: 4-AP, 4-aminopyridine; 3-AP, 3-aminopyridine; SE, status epilepticus; GABA, gamma-aminobutyric acid; KA, kainic acid; IPSP, inhibitory postsynaptic potential; EPSP, excitatory postsynaptic potential
} 

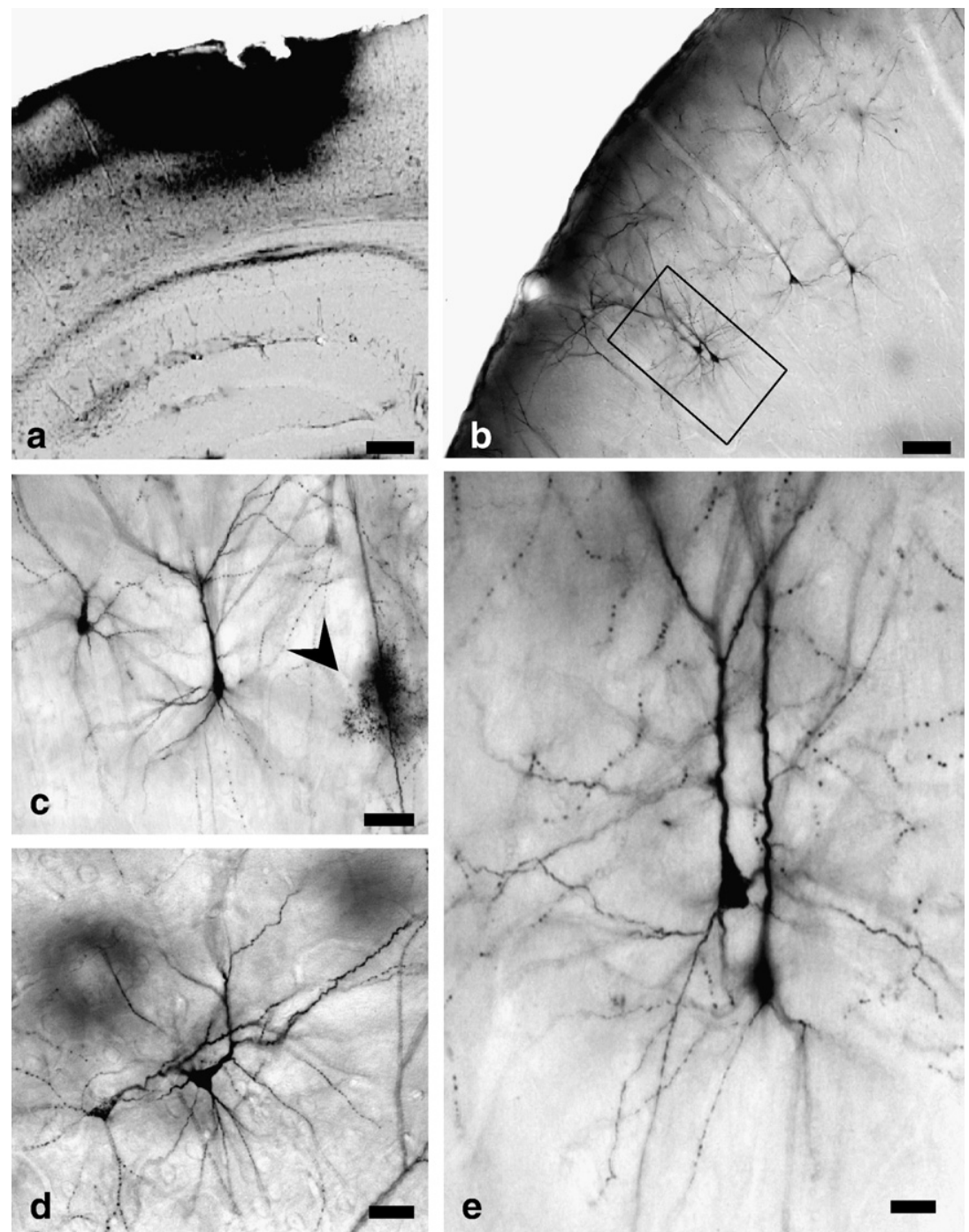

Fig. 1 - Light microscopic images of silver-stained "dark" neurons at the end of the 4-AP treatment (0-hour survival). Parietal cortex and hippocampus under the site of the 4-AP application site (a). Sporadic appearance of "dark" pyramidal cells in a distant cortical area (b). High power magnifications of "dark" pyramidal cells in the parietal (c and e) and the piriform (d) cortex. In c, an arrowhead points to a "dark" astrocyte. Scale bars: $a$ and $b=200 \mu \mathrm{m} ; c$ and $d=20 \mu \mathrm{m} ; \mathrm{e}=10 \mu \mathrm{m}$.

called "dark" (Agardh et al., 1981; Czurko and Nishino, 1993; Gallyas et al., 2004; Gallyas et al., 1992; Kellermayer et al., 2006; Poirier et al., 2000). The same kind of ultrastructural compaction can also be found in "dark" astrocytes (Gallyas et al., 1994; Toth et al., 1997) and in "dark" axons (Gallyas et al., 2002, 2006b; Gallyas and Zoltay, 1992).

A previous study from our laboratory (Slezia et al., 2004) used an ictal-interictal (3-AP) model to asses epileptic seizurerelated changes in the nucleoside micro-environment of hippocampal neurons after perfusion of 3-AP into the contralateral hippocampus via a microdialysis probe. In that study, no cell death but the disappearance of $\mathrm{Ca}^{++}$-binding proteins from hippocampal interneurons was observed. In the present study, we placed a small 4-aminopyridine (4-AP) crystal onto the exposed cortical surface. The 4-AP is a more selective potassium channel blocker than 3-AP and is known to cause electrographic seizures (Mihaly et al., 1990; Szente and Baranyi, 1987).
To visualize the neurons injured by our 4-AP epileptic seizure model, we used a silver staining method that is a selective and sensitive marker of "dark" neurons (Gallyas et al., 1990) and, in contrast to the traditional silver staining methods, gives reproducible results (Newman and Jasani, 1998). We also made EEG recordings, to look for a possible correlation between patterns of the epileptic seizure activity and the distribution of the accompanying morphological cell injury in space and time.

\section{Results}

To evoke seizure activity, a small crystal of the $\mathrm{K}^{+}$-channel blocker 4-AP was placed onto the exposed right parieto-occipital cortex of anesthetized rats for $40 \mathrm{~min}$. At three survival times (0, 3 and $6 \mathrm{~h}$ ), the presence of "dark" neurons and "dark" astrocytes was demonstrated by a silver staining method. The behavioral 

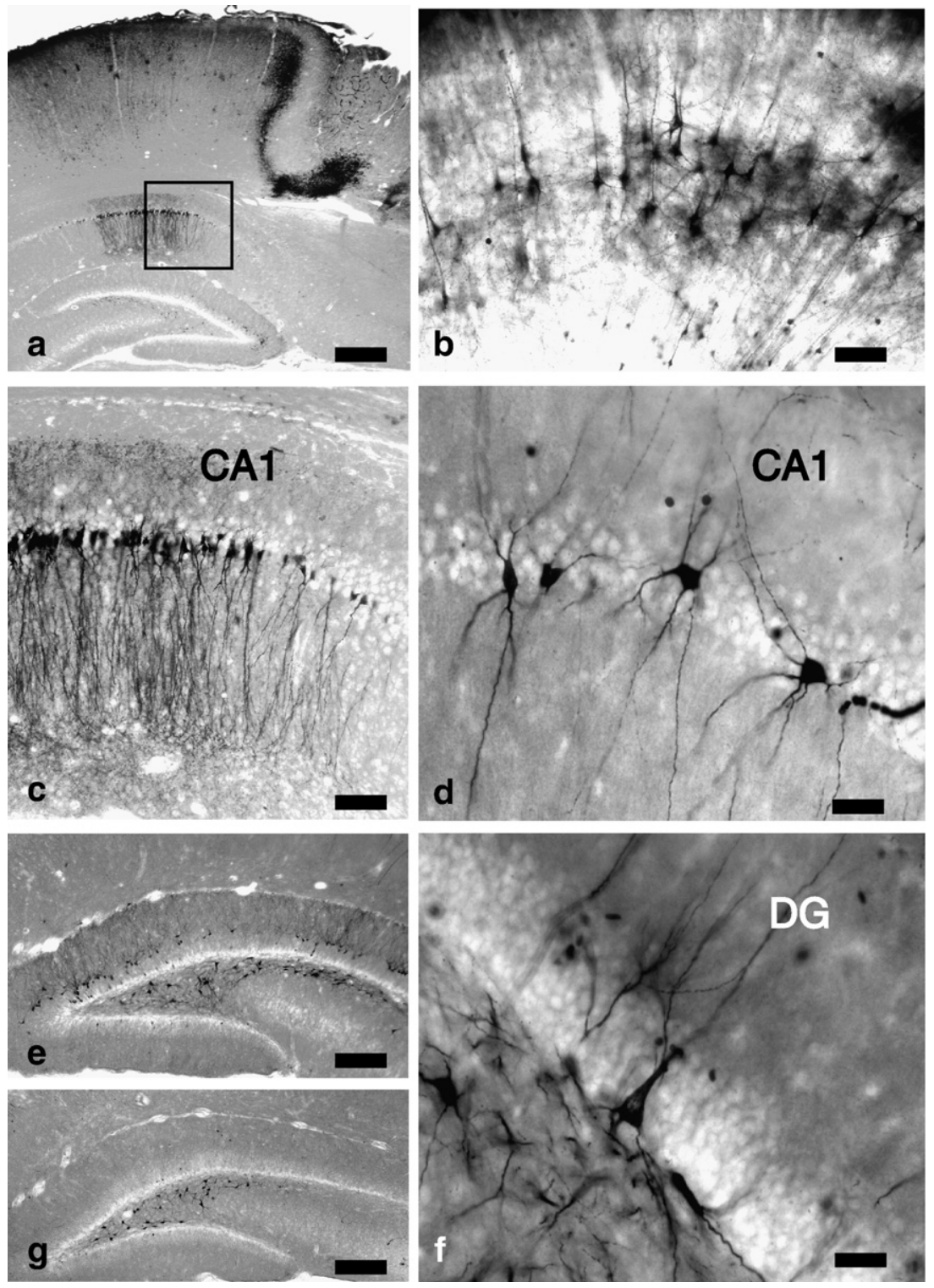

Fig. 2 - "Dark" cells in the parietal cortex and in the hippocampal areas at 3-hour survival. Sporadic "dark" pyramidal cells in cortical areas distant from the application site ipsilaterally (a) and contralaterally (b). In a, the boundary zone of the seriously damaged cortical area under the site of the 4-AP crystal application is stained black with silver. (c) High magnification of the ipsilateral CA1 region boxed in (a). (e) "dark" granule cells in the ipsilateral dentate gyrus. (d) "dark" interneurons in the ipsilateral CA1 region and $(\mathrm{f})$ in the dentate gyrus. (e) "Dark" interneurons in the ipsilateral hilus and (g) in the contralateral hilus. Scale bars: $a=400 \mu \mathrm{m} ; \mathrm{b}$ and $\mathrm{c}=\mathbf{1 0 0} \mu \mathrm{m} ; \mathrm{d}$ and $\mathrm{f}=\mathbf{2 0} \mu \mathrm{m} ; \mathrm{e}$ and $\mathrm{g}=\mathbf{2 0 0} \mu \mathrm{m}$.

signs of the epileptic activity were scored in rats with longer survival times ( $3 \mathrm{~h}$ and $6 \mathrm{~h}$ ). Nine rats (1 with 3-hour and 8 with 6 hour survival time) were mounted with skull-attached EEG electrodes one week before the 4-AP treatment. These rats had individual identity labels (ApF-1,-3,-5,-6 and ApF-A-ApF-E) and referred this way in the text and figures.

\subsection{Control rats}

No pathologic features were revealed by the silver staining method in the brain of the non-operated and sham-operated control rats in which the dura remained intact. In other shamoperated rats, in which the dura mater was opened, a small number of silver-stained ("dark") neurons were observed in the superficial layers of the cortex under the dura opening, irrespective of the survival time.

\subsection{Distribution of "dark" neurons at $0 \mathrm{~h}$ after the end of the 4-AP application}

In each of the four rats examined, the silver staining method revealed a dark black area in the parieto-occipital cortex under the site of the 4-AP crystal application (Fig. 1a). In this area, most cellular elements were injured, like in the case of any other kind of focal brain injuries (e.g. ischemia, Czurko and Nishino, 1993). In the cortical areas distant from the crystal 

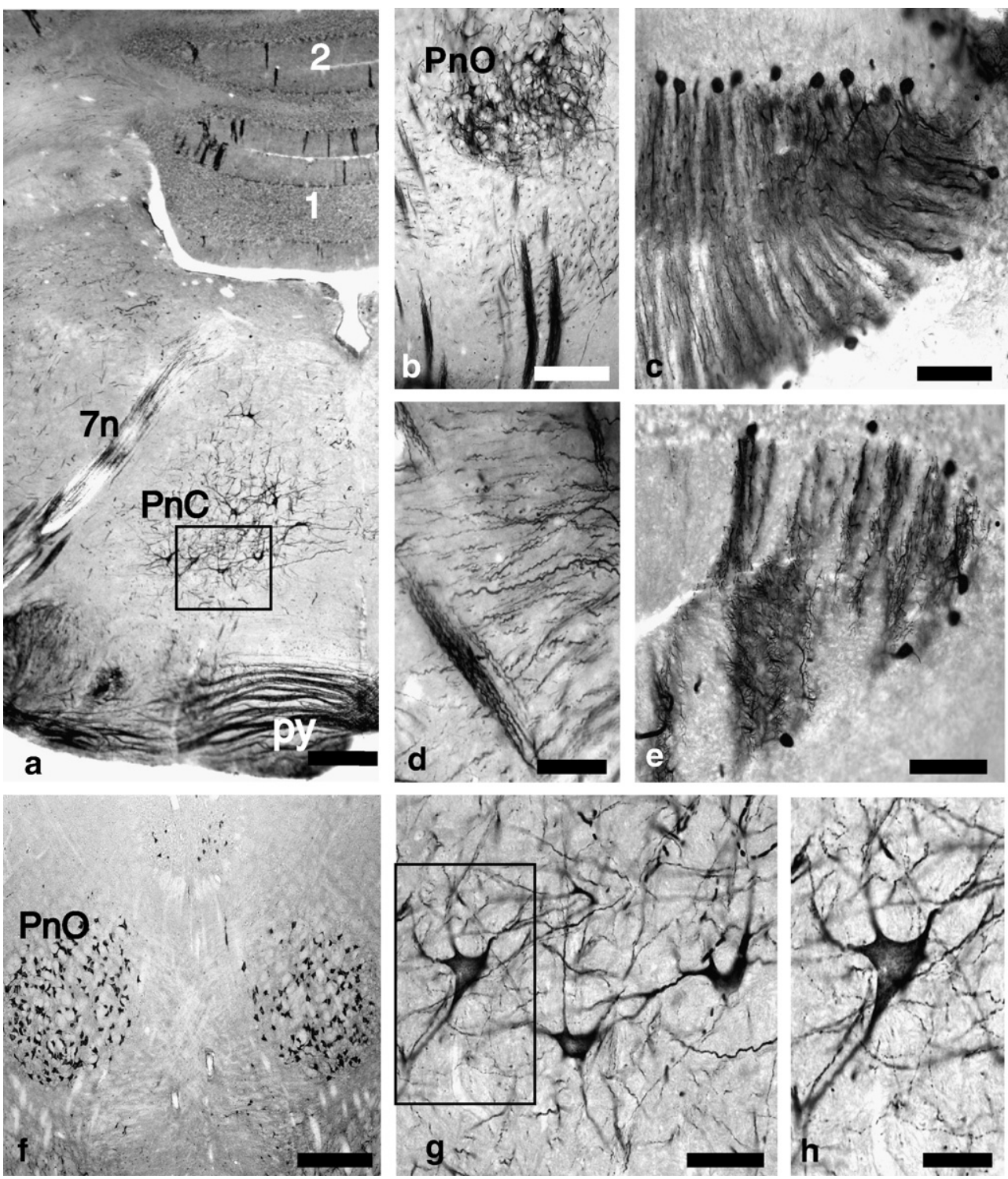

Fig. 3 - "Dark" neurons (a and f-h), "dark" axons (a, b and d) and "dark" Purkinje cells (c and e) in the pontine reticular formation at 3-hour (a-e, ApF-5) and 6-hour (f, ApF-1) survival. (a) Border zone of the medulla pons. "Dark" axons in the facial nerve root and in pyramidal tract are denoted with $7 \mathrm{n}$ and py, respectively, "dark" giant neurons in the caudal part of the pontine reticular nucleus with PnC, and "dark" Purkinje cells in the cerebellar lobules with 1 and 2. (b and d) "Dark" axon bundles in the rubrospinal tract. (c and e) "Dark" Purkinje cells in cerebellar lobules. (f, ApF-1) "Dark" neurons in the oral parts of the pontine reticular nuclei (PnO). (g) High magnification of the area boxed in a. (h) High magnification of the area boxed in g. ApF-1 and ApF-5 are the identity marks of the affected rats. Scale bars: $a, b$ and $f=400 \mu \mathrm{m} ; \mathrm{c}$ and $\mathrm{e}=100 \mu \mathrm{m} ; \mathrm{d}$ and $\mathrm{g}=60 \mu \mathrm{m} ; \mathrm{h}=30 \mu \mathrm{m}$.

application, in both the ipsilateral and in the contralateral cortices, sporadic appearance of Golgi-like (stained together with the dendritic arborization) pyramidal cells were observed (Figs. 1b-e), mainly in the superficial layers (layers II-III) of the ipsilateral parietal cortex (Figs. 1b, c, e) and the ipsilateral piriform cortex (Fig. 1d). In some cases, "dark" astrocytes were observed in the vicinity of "dark" neurons (arrowhead in Fig. 1c). In two rats, the contralateral frontal and parietal cortical areas contained sporadic "dark" pyramidal cells. In three rats, the hilus of the hippocampus contained sporadic "dark" neurons bilaterally, while sporadic "dark" hippocampal CA1 pyramidal cells were seen in one rat. Neither "dark" neurons nor "dark" astrocytes were present in the ponto-cerebellar brain areas of the two rats examined.

\subsection{Distribution of "dark" neurons at $3 \mathrm{~h}$ after the end of the 4-AP application}

In each of the five rats examined, the neuronal argyrophilia disappeared in the right parieto-occipital cortex under the site of the 4-AP crystal (Fig. 2a). The boundary of this area stained black with silver (Fig. 2a). The numbers of "dark" neurons in both the ipsi- and the contralateral parietal and frontal cortical areas were high. In two rats, the ipsilateral cortical areas were 

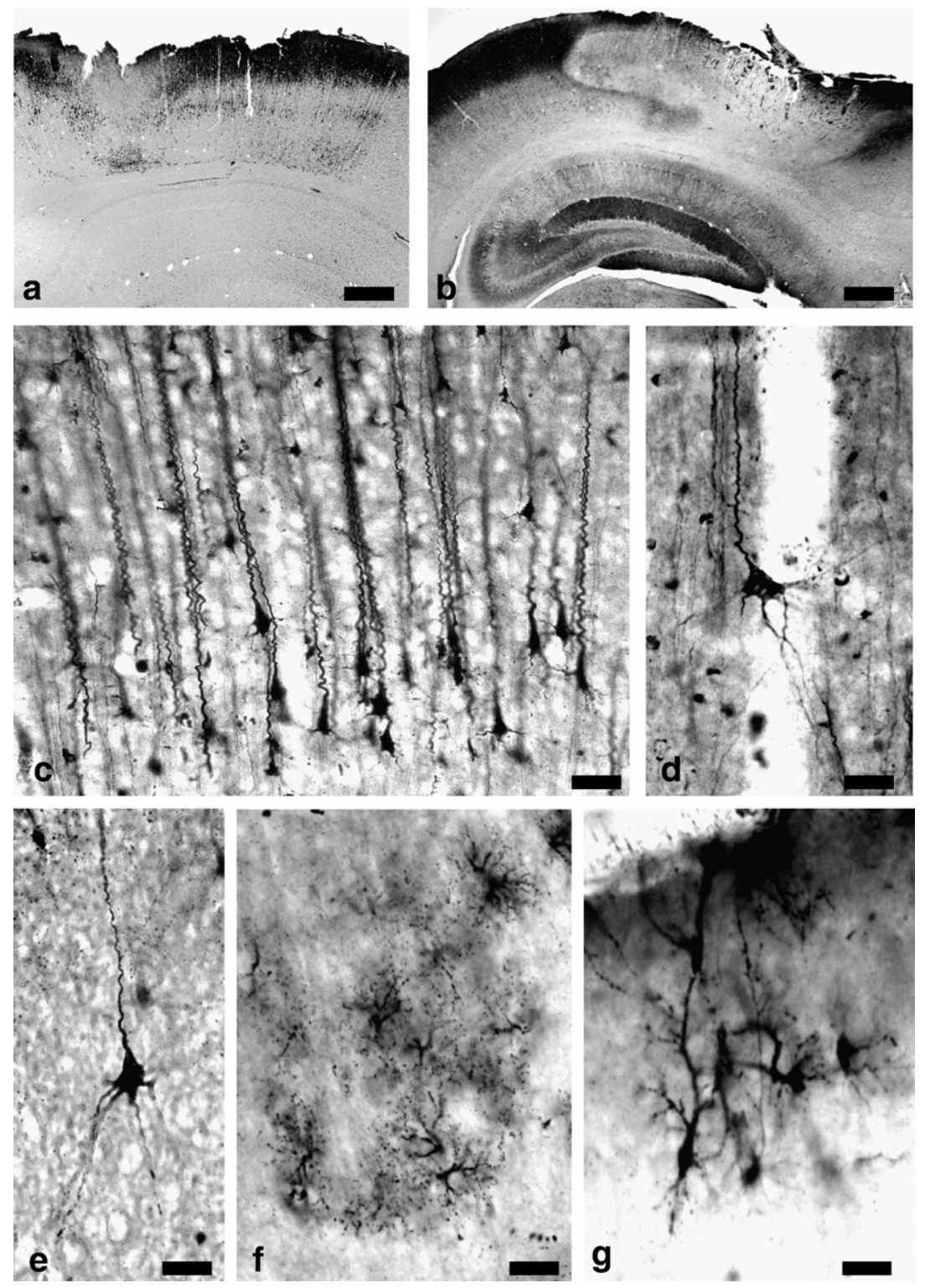

Fig. 4 - Silver stained "dark" cells at 6-hour survival. ( $a$ and b) Low and high incidence, respectively, of "dark" neurons in the parieto-occipital cortex and the hippocampus. (c-e) High magnifications of the parieto-occipital cortices demonstrated in b and a. (f) "Dark" astrocytes in the cortical area under the 4-AP crystal application. (g) Neurogliaform cells in the parietal cortex contralateral to the site of the 4-AP crystal application. Scale bars: $a$ and $b=500 \mu \mathrm{m} ; c=60 \mu \mathrm{m} ; d-f=30 \mu \mathrm{m} ; g=10 \mu \mathrm{m}$.

more affected. Sporadic "dark" pyramidal neurons were observed in both the dorsal and the ventral part of the hippocampus. The hippocampal involvement was nearly symmetrical, with the exception of one rat. "Dark" interneurons were seen in the CA1 pyramidal layer of the hippocampus (Fig. 2d), in the granule layer of the dentate gyrus (Fig. 2f) and in the hilus (Figs. 2e, g). The occurrence of "dark" interneurons in the hippocampal areas was more pronounced ipsilaterally. "Dark" astrocytes were found in all rats.

The ponto-cerebellar brain areas were examined in two rats. One of these (ApF-5) showed numerous "dark" neurons (Figs. 3a, f-h), "dark" axons (Figs. 3a, b and d) and "dark" Purkinje cells (Figs. 3c, e). In the other, a few giant "dark" neurons were found in the caudal part of the pontine reticular nucleus.
2.4. Distribution of "dark" neurons at $6 \mathrm{~h}$ after the end of the 4-AP application

In each of the 10 rats examined, the cortical areas close to the application site contained as much or more "dark" neurons than those which survived for $3 \mathrm{~h}$. In 5 of these rats, the numbers of "dark" neurons in the different brain areas decreased substantially. In two of them, there were hardly any "dark" neurons but a few neurogliaform cells (Kalinichenko et al., 2006) in the contralateral parietal cortical areas (Fig. 4g). In the other three rats (ApF-6, ApF-B and ApF-C), mostly the ipsilateral dorsal hippocampal areas contained "dark" neurons, but these were less in numbers than those found in the rats with 3-hour survival time (see the " $6 \mathrm{~h}$ " group 

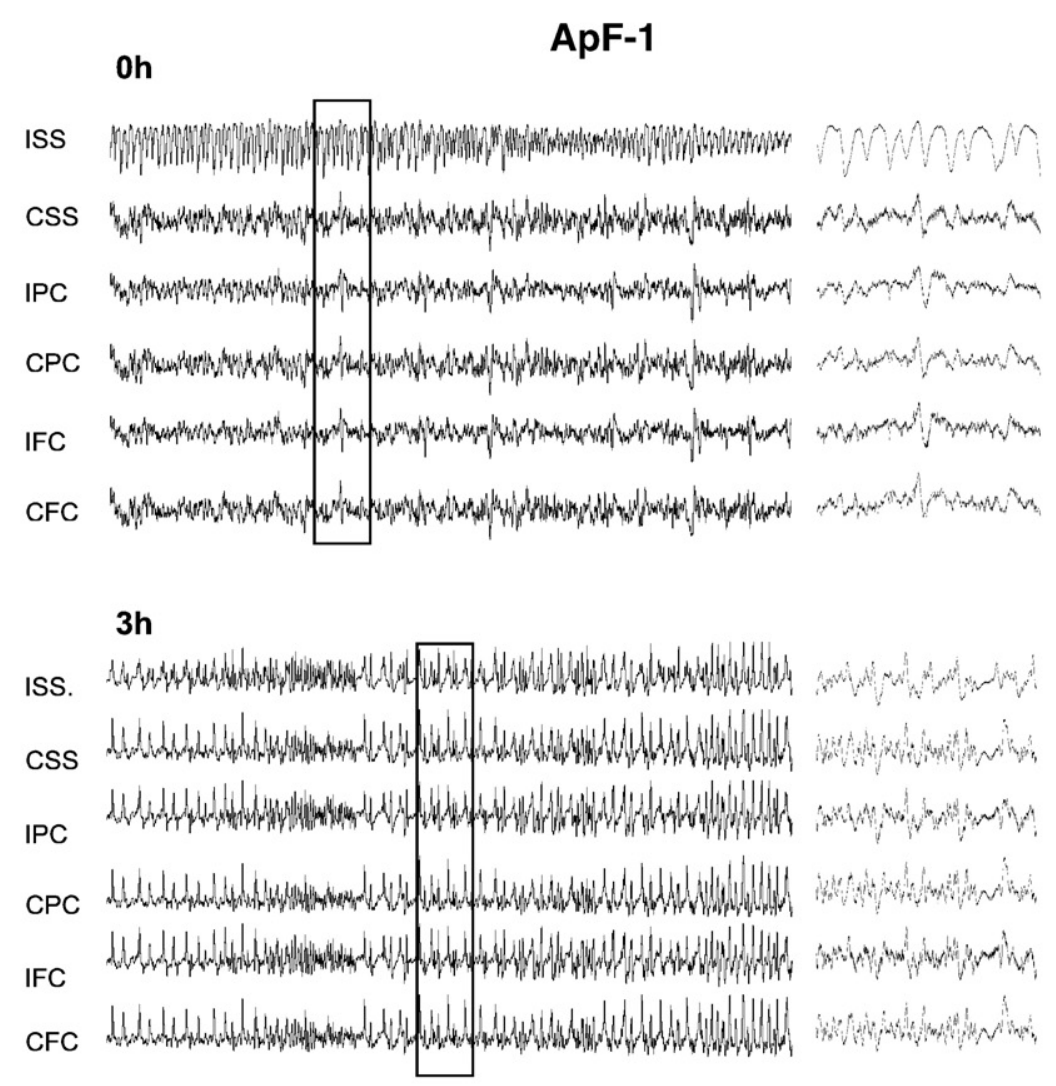

$6 h$

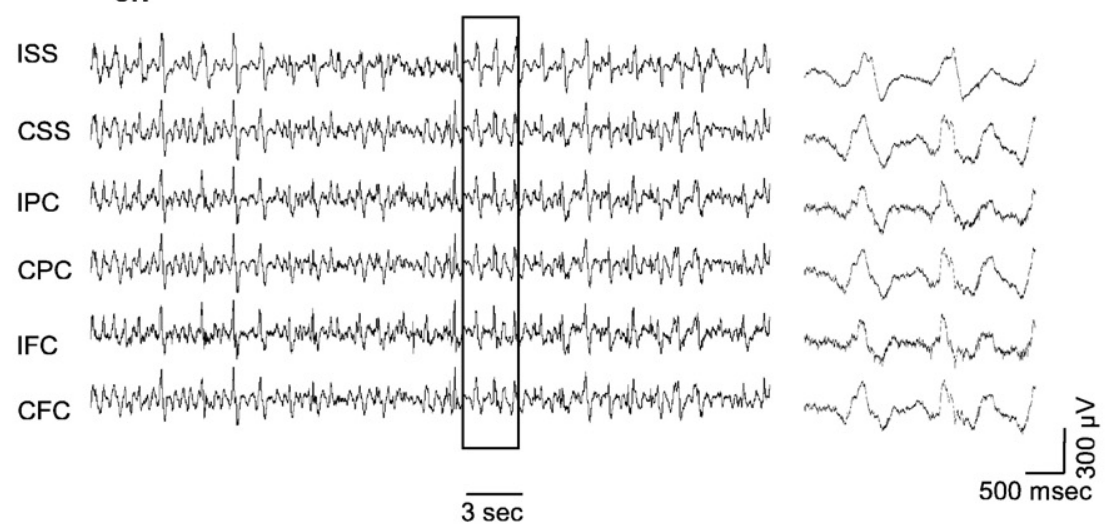

Fig. 5 - EEG activity periods demonstrating the progression of the epileptic activity in a rat (ApF-1) at $0 \mathrm{~h}, 3 \mathrm{~h}$ and $6 \mathrm{~h}$ after the 4-AP application. Higher temporal resolutions of the boxed areas are shown on the right. Abbreviations: IFC and CFC: ipsi- and contralateral frontal cortex, IPC and CPC: ipsi- and contralateral parieto-occipital cortex, ISS and CSS: ipsi- and contralateral somatosensory cortex.

in Fig. 7). "Dark" interneurons were found in one rat in the contralateral hippocampal areas (see Supplementary Table 1). In all three rats, giant "dark" neurons were observed in the pontine reticular formation (Fig. 7, Supplementary Fig. 1 and Table 1).

In the remaining five rats with 6-hour survival time (ApF-1, ApF-3, ApF-A, ApF-D and ApF-E), the occurrence of "dark" neurons was comparable to that of the rats with 3-hour survival time (see the "6 h-gen" group in Fig. 7). In these rats, the cortical areas contained individual "dark" pyramidal cells with markedly shrunken somata and corkscrew-like apical dendrites (Figs. 4c-e; ApF-1). Furthermore, both the ipsilateral and the contralateral hippocampal areas showed "dark" neurons both dorsally and ventrally (Fig. 7). "Dark" interneurons were found in four rats. In two of them, these were observed predominantly in the contralateral hippocampal areas (see Supplementary Table 1). "Dark" astrocytes (Fig. 4f) were observed under the site of the 4-AP crystal application (Fig. 4d). In four rats of this group, the ponto-cerebellar brain areas were affected, both the oral (PnO) and the caudal (PnC) parts of the pontine reticular nucleus were heavily and symmetrically involved (Fig. 3f, "6 h-gen" group in Fig. 7). 

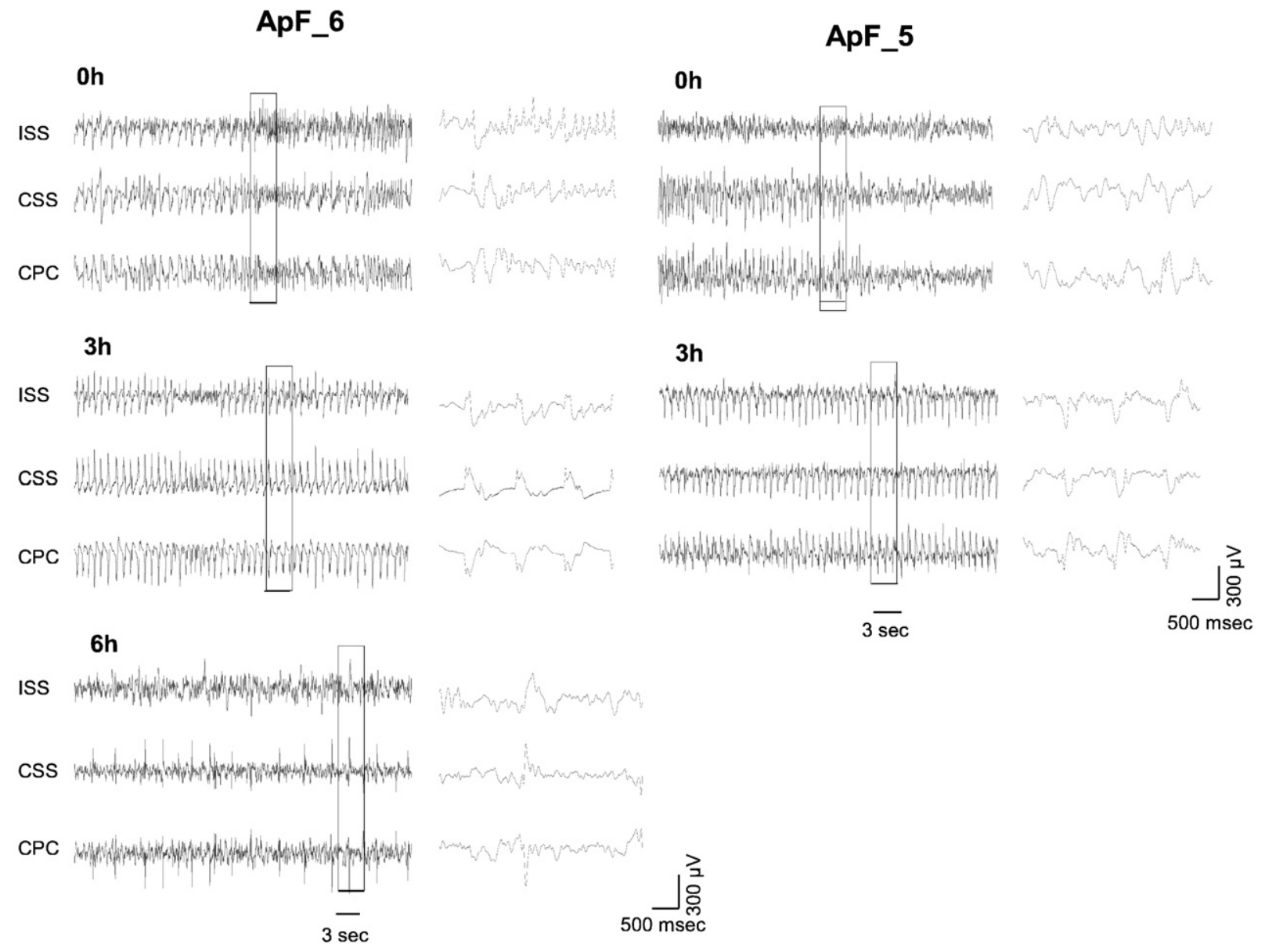

Fig. 6 - EEG activity periods demonstrating the temporal change of the epileptic activity in two rats, one of which (ApF-6) recovered from generalized seizures, the other survived for $3 \mathrm{~h}$. Abbreviations: IFC and CFC: ipsi- and contralateral frontal cortex, IPC and CPC: ipsi- and contralateral parieto-occipital cortex, ISS and CSS: ipsi- and contralateral somatosensory cortex.

\subsection{Behavioral manifestation of the epileptic activity}

The behavioral manifestation of the seizure activity was scored according to the Racine Scale. Within $30 \mathrm{~min}$ after the Halothane anesthesia had been discontinued, all rats reached Stage- 5 seizures. At $3 \mathrm{~h}$, all rats displayed Stage- 5 seizures and were in status epilepticus (SE). Out of the 10 rats examined at $6 \mathrm{~h}$, five (ApF-1, ApF-3, ApF-A, ApF-D and ApF-E) were still in Stage-5 seizure, in SE, two recovered from Stage-5 to Stages 12 and three (ApF-6, ApF-B and ApF-C) recovered from Stage-5 to Stages 2-3.

\subsection{Electrophysiology, EEG patterns}

During the first hour of EEG recording $(0 \mathrm{~h})$, the ictal EEG activity consisted of diverse patterns: small amplitude (10$20 \mu \mathrm{V})$ slow-repetitive-spikes (5-8 Hz), high amplitude (300$350 \mu \mathrm{V})$ slow-repetitive-spikes (5-8 Hz), high amplitude (250$350 \mu \mathrm{V})$ fast-repetitive-spikes (10-12 Hz) and high amplitude (300-350 $\mu \mathrm{V}$ ) low-frequency-spikes $(2-3 \mathrm{~Hz})$. The duration of the ictal periods varied from 5 to $20 \mathrm{~s}$ (see Figs. 5 and 6 , at $0 \mathrm{~h}$ ).

Three hours after the 4-AP application ( $3 \mathrm{~h}$ ), the EEG showed a much more homogenous pattern. Interictal periods almost completely disappeared from the recording and high amplitude slow-repetitive-spike activity dominated all channels of the EEG (generalized seizure activity and SE). The frequency of these spikes was 1.5-2 Hz, with amplitude of 300$350 \mu \mathrm{V}$ (see Figs. 5 and 6 at $3 \mathrm{~h}$ ).

Six hours after the 4-AP application, the 8 rats examined could be divided into two groups. In the five rats of the " $6 \mathrm{~h}$ gen" group, the EEG was similar to that of the $3 \mathrm{~h}$ recording, with sharper high amplitude generalized waves occurring with lower frequency (1-1.5 Hz) (see ApF-1 in Fig. 5; "6 h-gen" group in Fig. 7 and ApF-3 inSupplementary Fig. 1). In the three rats examined from the non-generalized $6 \mathrm{~h}$ group, the EEG recovered from the generalized seizures and the normallooking EEG was interrupted with narrow spikes (see ApF-6 in Fig. 6 and 6 h group in Fig. 7).

\subsection{Electrophysiology, Fourier analysis}

At $3 \mathrm{~h}$ after the 4-AP application, a peak at the low frequencies, around 1.5-2 Hz, was visible in the Power Spectral Density (PSD) graphs (ApF-5 in Fig. 7, Supplementary Fig. 2 at 3 h). At $6 \mathrm{~h}$, the three rats with non-generalized epilepsy (ApF-6, ApF-B and ApF-C in Fig. 7) had increased power in their PSD-s at the 


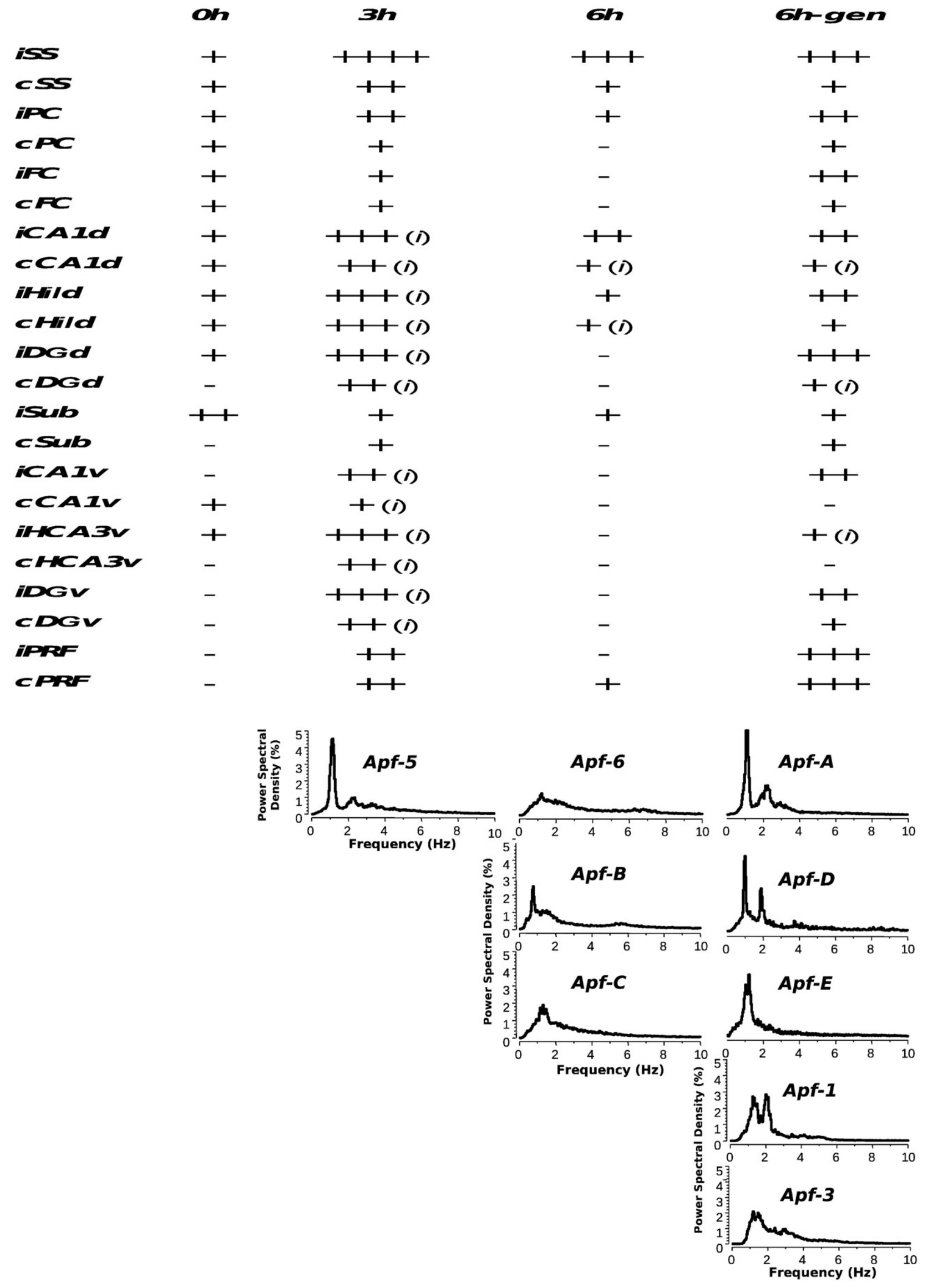


low frequencies, with less pronounced small peaks at $1-1.5 \mathrm{~Hz}$. Each rat with generalized epilepsy ( 6 h-gen in Fig. 7) had pronouncedly high peaks in their PSD at $1 \mathrm{~Hz}$ and in three of them there was an additional smaller peak at $2 \mathrm{~Hz}$. In one rat (ApF-3), there was only a slight increase in the PSD at the low frequencies with less pronounced peak at $1.5 \mathrm{~Hz}$ although its EEG contained generalized narrow spikes at around $1 \mathrm{~Hz}$ (seeSupplementary Fig. 1, ApF-3 at 6 h).

2.8. Correlation of the behavioral, electrophysiological and morphological findings

At $3 \mathrm{~h}$ all rats displayed Stage-5 seizures, the EEG-records showed generalized electric seizures (Figs. 5, 6 and Supplementary Fig. 2 at $3 \mathrm{~h}$ ), and "dark" neurons were present in the cortical areas, in both the dorsal and the ventral hippocampus and in the ponto-cerebellar brain areas. In the hippocampal areas, "dark" interneurons were seen.

At $6 \mathrm{~h}$, in those of the five rats that did not show generalized seizures, the number of "dark" neurons decreased substantially. In the two rats that recovered to Stages 1-2 seizures, there were very few "dark" neurons. In the three rats that recovered to Stages 2-3 seizures (ApF-6, ApF-B and ApF$C)$, there were "dark" neurons in the ipsilateral side of dorsal hippocampus and subiculum. "Dark" interneurons were found in the contralateral dorsal hippocampus in one rat (ApF-6 inSupplementary Table 1). In these rats, giant "dark" neurons were found in the pontine reticular formation (ApF-6, ApF-B and ApF-C inSupplementary Table 1). Note, that the EEG and the PSD analysis revealed intense generalized electric seizures at $3 \mathrm{~h}$ but not at $6 \mathrm{~h}$ in these rats (Supplementary Fig. 2).

In the five rats that had generalized seizures at $6 \mathrm{~h}(6 \mathrm{~h}$-gen group in Fig. 7; ApF-1, ApF-3, ApF-A, ApF-D and ApF-E inSupplementary Fig. 2 and Table 1) numerous "dark" neurons were present both in the cortical and the hippocampal areas and in the pontine reticular formation. However, there were differences between the individual rats. In two rats, the presence of "dark" neurons was less intensive in the cortical and hippocampal areas, but the pontine reticular formation was heavily involved. "Dark" interneurons were found in four out of these five rats, mostly in the contralateral dorsal hippocampal areas. Except for 1 rat, the pontine reticular formation was symmetrically affected (ApF-3). This rat had no "dark" neurons in the pontine reticular formation and, although it had monotone narrow spikes (50-60 ms at half amplitude) with $1 \mathrm{~Hz}$ in its EEG, it had a smaller peak in its PSD at $1.5 \mathrm{~Hz}$ (see ApF-3 in Fig. 7; Supplementary Figs. 1, 2 and Table 1). Interestingly, this rat had numerous "dark" interneurons in the contralateral dorsal hippocampal areas (Supplementary Table 1).

\section{Discussion}

\subsection{Potential of the 4-AP in epilepsy research}

The 4-AP is known to cause electrographic seizures when topically applied to the exposed neocortex (Mihaly et al., 1997; Szente and Baranyi, 1987), or tonic-clonic seizures when injected systemically (Mihaly et al., 1990). An advantage of the 4-AP models is that they do not cause extensive neuronal damage (Pena and Tapia, 2000; Slezia et al., 2004). Furthermore, 4-AP poisoning induces similar seizures in humans (Spyker et al., 1980; Thesleff, 1980).

Electrophysiologically, the 4-AP effectively enhances both EPSPs and IPSPs, blocks the voltage-dependent potassium conductance, prolongs the duration of the action potentials and thus promotes calcium entry (Szente and Baranyi, 1987; Thesleff, 1980). During electrographic seizures and sustained depolarization, the elevated intracellular $\mathrm{Ca}^{++}$can result in both pathological and protective changes in the cellular protein composition of neurons or glial cells. These changes in the cellular proteom are under intense investigation in epilepsy research (Eun et al., 2004; Greene et al., 2007; Jiang et al., 2007; Junker et al., 2005; Ryu et al., 2007; Yang et al., 2006). Some of the available results emphasize the role of cytoskeletal impairment and cytoskeletal rearrangement in epilepsy (Greene et al., 2007; Ryu et al., 2007; Yang et al., 2006).

\subsection{Potential of the "dark"-neuron phenomenon in epilepsy research}

Epileptic seizures could produce a "spectacular" morphological change in neurons (Soderfeldt et al., 1983). The affected neurons, which are randomly distributed among normallooking neurons frequently in an undamaged environment, are traditionally called "dark" (Covolan and Mello, 2000; Covolan and Mello, 2006; Gallyas et al., 1990; Hajnal et al., 1997; Mello and Covolan, 1996; Poirier et al., 2000). The morphological change in question consists in the dramatic

Fig. 7 - Distribution of the "dark" neurons in four rat groups and the power spectral densities (PSD) of individual rats made from the last one-hour EEG records. The relative numbers of "dark" neurons averaged from five $60-\mu \mathrm{m}$ sections of the structures listed in the first column are expressed with a semi-quantitative grading scale as follows: + means 1-5 "dark" neurons; ++ means 5-15 "dark" neurons, +++ means 15-30 "dark" neurons. Abbreviations: isS: ipsilateral somatosensory cortex; cSS: contralateral somatosensory cortex; iPC: ipsilateral parietal cortex; cPC: contralateral parietal cortex; iFC: ipsilateral frontal cortex; cFC: contralateral frontal cortex; iCA1d: ipsilateral CA1 region in the dorsal hippocampus; cCA1d: contralateral CA1 region in the dorsal hippocampus; iHild: ipsilateral dorsal hilus; cHild: contralateral dorsal hilus; iDGd: ipsilateral dorsal dentate gyrus; cDGd: contralateral dorsal dentate gyrus; iSub: ipsilateral subiculum; cSub: contralateral subiculum; iCA1v: ipsilateral CA1 region in the ventral hippocampus; cCA1v: contralateral CA1 region in the ventral hippocampus; iHilCA3v: ipsilateral hilus-CA3 complex in the ventral hippocampus; cHilCA3v: contralateral hilus-CA3 complex in the ventral hippocampus; iDGv: ipsilateral ventral dentate gyrus; cDGv: contralateral ventral dentate gyrus; iPRF: ipsilateral pontine reticular formation; cPRF: contralateral pontine reticular formation. 
decrease in the distances between normal-looking ultrastructural elements (ultrastructural compaction) in the somadendrite domain. The ultrastructural compaction is thought to be caused by a substantial change in the protein-water-ion equilibrium in the neuronal soma-dendrite domain (for a review see Gallyas, 2007; Gallyas et al., 2004).

From a pathophysiologic perspective, it is important to know that "dark" neurons can recover (Csordas et al., 2003; Czurko and Nishino, 1993) or die. The pathophysiological circumstances existing acutely after the formation of "dark" neurons will determine whether they will recover or die (Gallyas et al., 2006a).

The progression of "dark"-neuron formation was examined with silver staining and Fluoro-Jade labelling following pilocarpine-induced SE (Poirier et al., 2000). In the first hours after the one-hour SE, a significantly higher proportion of "dark" neurons was demonstrated by the silver staining method also used here as compared with that labeled with Fluoro-Jade. This fact suggests that the silver staining method can reveal the earliest stage of the "dark"-neuronal damage. It is important to note, that the "dark" neurons probably represent only a small portion of the cells ("the tip of the iceberg") which are involved in the pathological processes without any visible morphological damage.

The SE-related cell injury will ultimately be reflected in the cellular proteome, but more rapidly in the protein conformations. In order to plan future proteomic studies dealing with the epileptic seizure-related cellular stress, one has to identify first the distribution of the affected neuron populations in space and time. To obtain such information, we combined behavioral and electrophysiological methods with the silver staining of "dark" neurons.

\subsection{Possible effects of the formation of "dark" interneurons in the hippocampus}

It is an unsolved problem, why the immunoreactivity of parvalbumin, a $\mathrm{Ca}^{++}$-binding protein, disappears temporarily from the soma and dendrites of hippocampal interneurons found in a KA-epilepsy study (Magloczky and Freund, 1995), and in our previous 3-AP epilepsy study (Slezia et al., 2004). In the present 4-AP epilepsy study, numerous interneurons in the hippocampus became "dark" (compacted). This observation suggests that there may be a correlation between the epilepsyinduced "dark" state of these interneurons and the epilepsyinduced temporary disappearance of calcium-binding proteins. Specifically, one of the morphological characteristics of "dark" neurons is the disappearance of demonstrability of certain antigens (Gallyas, 2007).

Our observation that the "dark" interneurons appeared at first in the ipsilateral and later in the contralateral hippocampus supports the suggestion of Mihaly et al. (1997) that the inhibitory control could be more effective in the contralateral hemisphere than in the side of the 4-AP treatment (Mihaly et al., 1997).

\section{4. "Dark" neurons in the pontine reticular nuclei may be involved in the generalization of epileptic seizures}

Several electrophysiological studies have suggested that the pontine reticular formation participated in the generation and maintenance of the epileptic state (Elazar and Berchanski, 2000; Manjarrez et al., 2001; Peterson, 1995; Raisinghani and Faingold, 2005). Nevertheless, histopathological studies have not investigated this structure, probably because it is situated in the most caudal part of the rat brain. E.g., this area was not among the 53 brain areas that were investigated for the presence of "dark" neurons examined following pilocarpineor KA-induced SE (Covolan and Mello, 2000).

In the present study, different numbers of "dark" neurons were found in the pontine reticular nuclei in all but one rats that displayed generalized epileptic seizures (for at least $3 \mathrm{~h}$ ), depending on their Racine seizure score and EEG activity at $6 \mathrm{~h}$. In those which were constantly in generalized seizures and SE there were lots of "dark" neurons in this brain area, symmetrically on both sides. In these rats, the PSD analysis showed high peaks at the low frequencies ( 1 and $2 \mathrm{~Hz}$ ). In the rats which recovered to Stages 1-2 hardly any, while in those which recovered to Stages 2-3 an intermediate number of "dark" neurons were present.

The only rat that was in status epilepticus at $6 \mathrm{~h}$ without containing "dark" neurons in this brain area had a smaller peak in its PSD at $1.5 \mathrm{~Hz}$. Interestingly this rat had plenty of "dark" interneurons in the hippocampal areas. This observation, which suggests that the pontine reticular formation and the hippocampal interneurons are differently involved in the generalization of seizures, calls for supporting investigations.

\section{Experimental procedures}

\subsection{Animals}

Thirty adult male Sprague-Dawley rats (300-500 g) were kept in standard conditions having $12 \mathrm{~h}$ light-dark cycle and supplied with food and water ad libitum. Experiments were carried out on the basis of local ethical rules in accordance with the Hungarian Act of Animal Care and Experimentation (1998, XXVIII, section 243/1998), which is in conformity with the regulation of animal experiments in the European Community. All efforts were made to minimize pain and suffering and to reduce the number of animals used.

\subsection{Surgery procedures and EEG recordings}

Rats were anesthetized with a 1-1.5\% Halothane-air mixture and secured in a stereotaxic frame (David Kopf, USA). For the 4-AP application a hole (1.5 $\mathrm{mm}$ in diameter) was drilled into the skull above the right parieto-occipital cortex (A: $-6.2 \mathrm{~mm}$, L: $2.5 \mathrm{~mm}$; Paxinos, 1982 \#82 \}. In 10 rats for histology, the dura mater was carefully removed, and a piece of 4-AP crystal $(0.5 \mathrm{mg} / \mathrm{kg}$ ) was locally placed onto the cortex. The hole was covered with a piece of artificial fibrin sponge. Forty minutes thereafter, the hole was washed out with physiologic saline, covered with bone wax and the Halothane anesthesia was discontinued. Four rats were sacrificed immediately, 4 survived for $3 \mathrm{~h}$ and 2 for $6 \mathrm{~h}$. Three non-operated and 6 shamoperated rats served as control. In the latter, the whole surgery procedure was done except for the 4-AP crystal application. Two of these rats were immediately fixed transcardially ( $0 \mathrm{~h}), 2$ after a 3-hour delay and 2 after a 6-hour delay. 
In the 9 rats for both histology and EEG recordings, 6 holes were drilled into the superficial layer of the skull above the frontal, the somatosensory and the parieto-occipital cortices bilaterally; each was filled with conductive paste, through which a stainless-steel electrode was inserted. An indifferent silver plate electrode was placed under the skin with the inner side covered with dental acrylic. The 6 electrodes and a multichannel connector were embedded in dental acrylic together with a rod ( $2 \mathrm{~mm}$ in diameter), the removal of which provided a channel for the future 4-AP application. One week later, a hole was drilled through this channel and the 4-AP crystal was placed onto the exposed cortical surface. Forty minutes after the 4-AP application, the hole in the skull was washed out with physiologic saline and covered with bone wax. Thereafter, the Halothane anesthesia was discontinued and the EEG recording was started. One rat was sacrificed $3 \mathrm{~h}$ later, the other 8 rats $6 \mathrm{~h}$ later. For control, 2 rats were operated on as described above, except the 4-AP crystal application. They survived for $3 \mathrm{~h}$ and $6 \mathrm{~h}$.

The EEG activity was recorded by a Grass EEG 8B model, filtered at $0.3 \mathrm{~Hz}$ to $70 \mathrm{~Hz}$ band width and the amplification was $7 \mu \mathrm{V} / \mathrm{mm}$. Data collection was performed with a CED 1401 data processing system using SPIKE2 v2.1 software from CED (Cambridge, UK). The sampling rate was 3000 Hz. Sixtyminute samples were stored and the Fourier Power Spectral Density (PSD) was calculated by NeuroExplorer v.3.2 (Nex Technologies, MA, USA).

\subsection{Behavioral scoring, EEG recording and data processing}

To score the behavior of the rats after 4-AP administration the Racine Scale was used (Racine, 1972) with modifications to the present seizure model (Malhotra and Gupta, 1997; Medina-Ceja et al., 2008). Specifically, Stage-0 means behavioral arrest (motionless), hair raising, excitement and rapid breathing; Stage-1 means salivation and unilateral movement of the lips, tongue and vibrissae; Stage- 2 means head nodding, head and eye clonus; Stage-3 means unilateral or bilateral forelimb clonus and "wet dog shakes"; Stage-4 means forelimb clonic seizures and clonic rearing; Stage-5 means generalized clonic seizures with falling, uncontrollable jumping and atonia.

\subsection{Perfusion and tissue sectioning}

Both 4-AP treated and control rats were deeply anesthetized with an overdose of urethane (2 g/kg i.p.), and perfused through the aorta with physiological saline followed by a fixative containing $4 \%$ paraformaldehyde in cacodylate buffer (Gallyas et al., 1993). Brains were removed from the skull 1 day later, then immersed in the same fixative for 1-3 days and frozen-sectioned at $60 \mu \mathrm{m}$. Every fifth of them was processed for silver staining (Gallyas et al., 1990, 1993). The remaining sections were stored in the fixative containing $0.01 \%$ sodium azide at $4^{\circ} \mathrm{C}$ for later use.

\subsection{Silver staining of "dark" neurons}

Sections were incubated for $16 \mathrm{~h}$ at $56^{\circ} \mathrm{C}$ in 1-propanol containing $1.2 \%$ sulfuric acid (esterification). Following a 5 - minute treatment in $1 \%$ acetic acid, they were immersed in a silicotungstate physical developer until the background turned yellowish-brown. Development was terminated by washing in $1 \%$ acetic acid for $30 \mathrm{~min}$. Sections were dehydrated, mounted, embedded in DePex and coverslipped.

\section{Acknowledgments}

We thank J. Csicsvari, A. Hajnal and K.A. Kékesi for reviewing the manuscript. This work was supported by a Regional Center of Excellence grants: DNT RET and CellKom RET, Hungary. András Czurkó was supported by a HAS János Bolyai Research Fellowship during this project.

\section{Appendix A. Supplementary data}

Supplementary data associated with this article can be found, in the online version, at doi:10.1016/j.brainres.2008.06.044.

\section{R E F E R E N C E S}

Agardh, C.D., Kalimo, H., Olsson, Y., Siesjo, B.K., 1981. Reply to the remarks by J. B. Brierley and A. W. Brown. Acta. Neuropathol 55, 323-325.

Benardo, L.S., 1997. Recruitment of GABAergic inhibition and synchronization of inhibitory interneurons in rat neocortex. J. Neurophysiol 77, 3134-3144.

Covolan, L., Mello, L.E., 2000. Temporal profile of neuronal injury following pilocarpine or kainic acid-induced status epilepticus. Epilepsy Res 39, 133-152.

Covolan, L., Mello, L.E., 2006. Assessment of the progressive nature of cell damage in the pilocarpine model of epilepsy. Braz. J. Med. Biol. Res 39, 915-924.

Csordas, A., Mazlo, M., Gallyas, F., 2003. Recovery versus death of "dark" (compacted) neurons in non-impaired parenchymal environment: light and electron microscopic observations. Acta Neuropathol (Berl) 106, 37-49.

Czurko, A., Nishino, H., 1993. 'Collapsed' (argyrophilic, dark) neurons in rat model of transient focal cerebral ischemia. Neurosci. Lett 162, 71-74.

Elazar, Z., Berchanski, A., 2000. Excitatory amino acids modulate epileptogenesis in the brain stem. Neuroreport 11, 1777-1780.

Eun, J.P., Choi, H.Y., Kwak, Y.G., 2004. Proteomic analysis of human cerebral cortex in epileptic patients. Exp. Mol. Med 36, 185-191.

Gallyas, F., 2007. Novel cell-biological ideas deducible from morphological observations on "dark" neurons revisited. Ideggyogy Sz 60, 212-222.

Gallyas, F., Zoltay, G., 1992. An immediate light microscopic response of neuronal somata, dendrites and axons to noncontusing concussive head injury in the rat. Acta. Neuropathol. (Berl) 83, 386-393.

Gallyas, F., Guldner, F.H., Zoltay, G., Wolff, J.R., 1990. Golgi-like demonstration of "dark" neurons with an argyrophil III method for experimental neuropathology. Acta Neuropathol (Berl) 79, 620-628.

Gallyas, F., Zoltay, G., Dames, W., 1992. Formation of "dark" (argyrophilic) neurons of various origin proceeds with a common mechanism of biophysical nature (a novel hypothesis). Acta. Neuropathol. (Berl) 83, 504-509.

Gallyas, F., Hsu, M., Buzsaki, G., 1993. Four modified silver methods for thick sections of formaldehyde-fixed mammalian central nervous tissue: 'dark' neurons, perikarya of all neurons, 
microglial cells and capillaries. J. Neurosci. Methods 50, 159-164.

Gallyas, F., Horvath, Z., David, K., Liposits, Z., 1994. An immediate morphopathologic response of a subpopulation of astrocytes to electroshock: "dark" astrocytes. Neurobiology (Bp) 2, 245-253.

Gallyas, F., Farkas, O., Mazlo, M., 2002. Traumatic compaction of the axonal cytoskeleton induces argyrophilia: histological and theoretical importance. Acta Neuropathol (Berl) 103, 36-42.

Gallyas, F., Farkas, O., Mazlo, M., 2004. Gel-to-gel phase transition may occur in mammalian cells: mechanism of formation of "dark" (compacted) neurons. Biol Cell 96, 313-324.

Gallyas, F., Gasz, B., Szigeti, A., Mazlo, M., 2006a. Pathological circumstances impair the ability of "dark" neurons to undergo spontaneous recovery. Brain Res 1110, 211-220.

Gallyas, F., Pal, J., Farkas, O., Doczi, T., 2006b. The fate of axons subjected to traumatic ultrastructural (neurofilament) compaction: an electron-microscopic study. Acta. Neuropathol. (Berl) 111, 229-237.

Greene, N.D., Bamidele, A., Choy, M., de Castro, S.C., Wait, R., Leung, K.Y., Begum, S., Gadian, D.G., Scott, R.C., Lythgoe, M.F., 2007. Proteome changes associated with hippocampal MRI abnormalities in the lithium pilocarpine-induced model of convulsive status epilepticus. Proteomics 7, 1336-1344.

Hajnal, A., Lenard, L., Czurko, A., Sandor, P., Karadi, Z., 1997. Distribution and time course of appearance of "dark" neurons and EEG activity after amygdaloid kainate lesion. Brain Res. Bull 43, 235-243.

Jiang, W., Du, B., Chi, Z., Ma, L., Wang, S., Zhang, X., Wu, W., Wang, X., Xu, G., Guo, C., 2007. Preliminary explorations of the role of mitochondrial proteins in refractory epilepsy: some findings from comparative proteomics. J. Neurosci. Res 85, 3160-3170.

Junker, H., Spate, K., Suofu, Y., Walther, R., Schwarz, G., Kammer, W., Nordheim, A., Walker, L.C., Runge, U., Kessler, C., Popa-Wagner, A., 2005. Proteomic identification of the involvement of the mitochondrial Rieske protein in epilepsy. Epilepsia 46, 339-343.

Kalinichenko, S.G., Pushchin II, Dyuizen, I.V., 2006. Neurochemical diversity of neurogliaform cells in the human primary motor cortex. J. Chem. Neuroanat. 31, 304-310.

Kellermayer, R., Zsombok, A., Auer, T., Gallyas, F., 2006. Electrically induced gel-to-gel phase-transition in neurons. Cell. Biol. Int 30, 175-182.

Magloczky, Z., Freund, T.F., 1995. Delayed cell death in the contralateral hippocampus following kainate injection into the CA3 subfield. Neuroscience 66, 847-860.

Malhotra, J., Gupta, Y.K., 1997. Effect of adenosine receptor modulation on pentylenetetrazole-induced seizures in rats. Br. J. Pharmacol 120, 282-288.

Manjarrez, J., Alvarado, R., Camacho-Arroyo, I., 2001. Differential effects of NMDA antagonists microinjections into the nucleus reticularis pontis caudalis on seizures induced by pentylenetetrazol in the rat. Epilepsy Res 46, 39-44.

Medina-Ceja, L., Cordero-Romero, A., Morales-Villagran, A., 2008. Antiepileptic effect of carbenoxolone on seizures induced by 4-aminopyridine: a study in the rat hippocampus and entorhinal cortex. Brain Res 1187, 74-81.

Mello, L.E., Covolan, L., 1996. Spontaneous seizures preferentially injure interneurons in the pilocarpine model of chronic spontaneous seizures. Epilepsy Res 26, 123-129.

Mihaly, A., Bencsik, K., Solymosi, T., 1990. Naltrexone potentiates 4-aminopyridine seizures in the rat. J. Neural. Transm. Gen. Sect 79, 59-67.
Mihaly, A., Szente, M., Dubravcsik, Z., Boda, B., Kiraly, E., Nagy, T., Domonkos, A., 1997. Parvalbumin- and calbindin-containing neurons express c-fos protein in primary and secondary (mirror) epileptic foci of the rat neocortex. Brain Res 761, 135-145.

Mody, I., Otis, T.S., Staley, K.J., Kohr, G., 1992. The balance between excitation and inhibition in dentate granule cells and its role in epilepsy. Epilepsy. Res. Suppl 9, 331-339.

Newman, G.R., Jasani, B., 1998. Silver development in microscopy and bioanalysis: past and present. J. Pathol 186, 119-125.

Ogita, K., Okuda, H., Watanabe, M., Nagashima, R., Sugiyama, C., Yoneda, Y., 2005. In vivo treatment with the $\mathrm{K}+$ channel blocker 4-aminopyridine protects against kainate-induced neuronal cell death through activation of NMDA receptors in murine hippocampus. Neuropharmacology 48, 810-821.

Pena, F., Tapia, R., 2000. Seizures and neurodegeneration induced by 4 -aminopyridine in rat hippocampus in vivo: role of glutamate- and GABA-mediated neurotransmission and of ion channels. Neuroscience 101, 547-561.

Peterson, S.L., 1995. Infusion of NMDA antagonists into the nucleus reticularis pontis oralis inhibits the maximal electroshock seizure response. Brain Res 702, 101-109.

Poirier, J.L., Capek, R., De Koninck, Y., 2000. Differential progression of Dark neuron and Fluoro-Jade labelling in the rat hippocampus following pilocarpine-induced status epilepticus. Neuroscience 97, 59-68.

Racine, R.J., 1972. Modification of seizure activity by electrical stimulation. II. Motor seizure. Electroencephalogr Clin Neurophysiol. 32, 281-294.

Raisinghani, M., Faingold, C.L., 2005. Pontine reticular formation neurons are implicated in the neuronal network for generalized clonic seizures which is intensified by audiogenic kindling. Brain Res 1064, 90-97.

Ryu, M.J., Kim, D., Kang, U.B., Kim, J., Shin, H.S., Lee, C., Yu, M.H., 2007. Proteomic analysis of gamma-butyrolactone-treated mouse thalamus reveals dysregulated proteins upon absence seizure. J. Neurochem 102, 646-656.

Slezia, A., Kekesi, A.K., Szikra, T., Papp, A.M., Nagy, K., Szente, M., Magloczky, Z., Freund, T.F., Juhasz, G., 2004. Uridine release during aminopyridine-induced epilepsy. Neurobiol. Dis 16, 490-499.

Soderfeldt, B., Kalimo, H., Olsson, Y., Siesjo, B.K., 1983. Bicuculline-induced epileptic brain injury. Transient and persistent cell changes in rat cerebral cortex in the early recovery period. Acta. Neuropathol. 62, 87-95.

Spyker, D.A., Lynch, C., Shabanowitz, J., Sinn, J.A., 1980. Poisoning with 4-aminopyridine: report of three cases. Clin. Toxicol 16, 487-497.

Szente, M., Baranyi, A., 1987. Mechanism of aminopyridine-induced ictal seizure activity in the cat neocortex. Brain Res 413, 368-373.

Thesleff, S., 1980. Aminopyridines and synaptic transmission. Neuroscience 5, 1413-1419.

Toth, Z., Seress, L., Toth, P., Ribak, C.E., Gallyas, F., 1997. A common morphological response of astrocytes to various injuries: "dark" astrocytes. A light and electron microscopic analysis. J. Hirnforsch. 38, 173-186.

Yang, J.W., Czech, T., Felizardo, M., Baumgartner, C., Lubec, G., 2006. Aberrant expression of cytoskeleton proteins in hippocampus from patients with mesial temporal lobe epilepsy. Amino Acids 30, 477-493. 